\title{
Perfil lipídico neonatal vs. perfil lipídico placentario
}

\author{
José Henry Osorio O.;* Jarol Augusto Quintero G.**; Jhon Jairo Osorio O.**; Diego Fernando Meza M.**; Germán \\ Gutiérrez M.**
}

\begin{abstract}
RESUMEN
El presente trabajo fue realizado en las salas de parto del Hospital de Caldas en Manizales y se determinó si al momento de realizar exámenes de laboratorio que tengan que ver con los lípidos de cordón umbilical, puede hacerse la punción del lado neonatal o del lado placentario indistintamente. Se tomaron muestras de sangre mediante punción $(3 \mathrm{ml})$ para determinar el perfil lipídico en 56 nacimientos espontáneos normales, tanto del lado placentario, como del lado neonatal del cordón umbilical para realizar la comparación utilizando una prueba t de student. La determinación del perfil lipídico se realizó mediante el método enzimático-colorimétrico.
\end{abstract}

Encontramos diferencia altamente significativa $(\mathbf{p}<0.01)$ al analizar los niveles de triglicéridos de ambos lados y altamente significativa $(p<0.01)$ al comparar Colesterol VLDL. Por tal motivo recomendamos para hacer la determinación del perfil lipídico al momento de nacer, tomar la muestra del lado neonatal después de pinzado el cordón umbilical.

PALABRAS CLAVES: Perfil lipídico, neonato, placenta, sangre de cordón umbilical.

\section{SUMMARY}

This work was done in the Hospital de Caldas, Manizales, Colombia and it was research if at born taken samples of cord blood for lipid profile, may it be taken from neonatal side or from placental side. We took samples by punction for measuring the lipid profile in 56 normal spontaneous borns of two sides and results was compared by t student test. The lipid profile was done by enzimatic-colorimetric method.

We found high significance $(p<0.01)$ during comparation of tryglicerides and high significance $(p<0.01)$ in cholesterol's comparation. We are recommending for lipid profile at born, to take the samples in neonatal side after it is clamped.

KEY WORDS: Lipid profile, neonate, placenta, cord blood.

\section{Introducción}

La sangre de cordón umbilical, tradicionalmente se ha utilizado en obstetricia para determinar la presencia de bacterias por hemocultivo, o realizar hemoclasificación y Coombs directo.

Otros usos de la sangre de cordón umbilical en el diagnóstico clínico son los siguientes: lactato, glucosa, anticuerpos ante la sospecha de enfermedades infecciosas (TORCHS: Toxoplasma, rubeola, citomegalovirus, herpes virus, y sífilis), cuadro hemático, bilirrubina, y cariotipo.

En el caso especial de las medidas de lípidos de cordón umbilical, se ha observado en experiencias aisladas que los valores a ambos lados de la pinza colocada en cordón después del nacimiento difieren (1), pero no se ha comprobado experimentalmente si esa variación es significativa.

* D.V.M.B.Sc. M.Sc. M.Sc. Est. de Ph.D. U. Newcastle. Director de la línea de Investigación en Bioquímica y Salud Departamento de Ciencias Básicas de la Salud. Universidad de Caldas. Manizales, Colombia.

** $\quad$ M.D. Especialistas en Ginecología y obstetricia. Departamento Materno Infantil. Universidad de Caldas. Manizales, Colombia. M.D. B. Sc. Especialista en Educación Sexual, Residente III, niveles Ginecología y obstetricia.

** M.D. Residentes III nivel en Ginecología y Obstetricia.

\section{Pacientes y métodos}

Las determinaciones se hicieron en 56 muestras de nacimientos normales espontáneos, en las salas de parto del Hospital de Caldas. Como criterio de selección se utilizó una encuesta aplicada a las madres, buscando con ello seleccionar una población con el mínimo de riesgos de alteraciones en su perfil lipídico.

56 muestras tomadas mediante punción del muñón del lado neonatal $(3 \mathrm{ml})$, y 56 muestras tomadas del muñón del lado placentario, (dos muestras en cada parto) siendo un total de 112 muestras.

Fue extraído el suero de las muestras tomadas y mediante el método enzimático colorimétrico se determinó el perfil lipídico, utilizando reactivos de la casa Grunenthal. A los datos obtenidos se les aplicó una prueba t para analizar dos variables: Perfil lipídico del lado placentario y Perfil lipídico del lado neonatal.

\section{Resultados}

La comparación de los valores promedio entre los dos grupos puede apreciarse en la tabla 1 y la figura 1 . 
Tabla 1

COMPARACION DE LOS VALORES PROMEDIO DEL PERFIL LIPIDICO NEONATAL VS PERFIL LIPIDICO PLACENTARIO

\begin{tabular}{|lrrrrr|}
\hline Muestra & Col-Total & Col-HDL & TAG & Col- & Col-LDL \\
\hline No. & & & & VLDL & \\
Neonato & 56,9 & 10,3 & 37,5 & 7,4 & 38,3 \\
Placenta & 63,0 & 10,1 & 52,4 & 10,3 & 42,2 \\
\hline
\end{tabular}

Figura 1

COMPARACION DEL PERFIL LIPIDICO NEONATAL VS PERFIL LIPIDICO PLACENTARIO

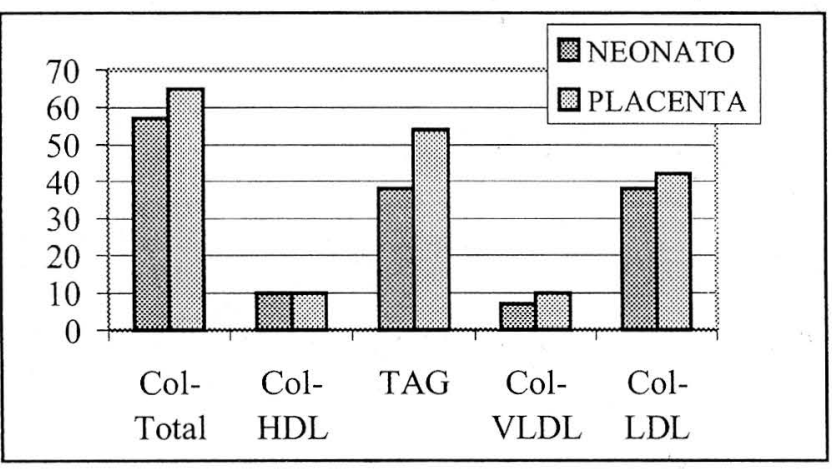

\section{Discusión}

La toma de muestras de cordón umbilical, es una de las prácticas más comunes en investigación y en la práctica clínica con múltiples propósitos.

Cuando se habla de toma de muestras de cordón umbilical, debe hacerse mención a situaciones como la introducción de la cordocentesis por Daffos y Cols. (2) la cual modificó espectacularmente el control y tratamiento de las pacientes sensibilizadas con incompatibilidad $\mathrm{Rh}$.

En este caso, las muestras sanguíneas permiten una medida precisa del hematocrito y de la concentración de hemoglobina fetal para determinar la gravedad del proceso hemolítico y la necesidad de una transfusión intrauterina. Además, esta misma técnica puede utilizar- se para la transfusión intravascular directa al feto (3) lo que ha permitido el tratamiento intrauterino con éxito de los fetos hidrópicos y que no responden a la transfusión en la cavidad peritoneal (4).

Para realizar gasometría en sangre de cordón umbilical, el American College of Obstetricians and Gynecologists (5) recomienda el doble pinzamiento del cordón, la separación de un segmento del mismo tras el nacimiento, que se coloca en la mesa y se emplea para determinar los gases, en caso de que cualquier anomalía importante en el proceso del parto, o la persistencia de problemas en la situación del neonato más allá de los primeros 5 minutos de vida lo hagan recomendable. Dentro de los programas de detección precoz de enfermedad coronaria, puede estudiarse el perfil lipídico en sangre de cordón, porque pueden registrarse en niños que no han ingerido alimentos, todos los contenidos lipídicos promedio en sangre, los cuales pueden ser correlacionados con los antecedentes genéticos, el estilo de vida de las madres durante el embarazo y las características del parto, para detectar dislipidemias congénitas o predecir el comportamiento lipídico en meses o años venideros (6).

Con miras a esclarecer, si en efecto, puede usarse o no sangre de cualquier lado del cordón umbilical, para tal fin realizamos el presente estudio, encontrando en las muestras de cordón umbilical del lado neonatal, valores promedio que están acordes por los reportados por Osorio y cols. en 1997 (7), detectando a la vez una diferencia altamente significativa $(\mathrm{p}<0.01)$ entre los valores de triglicéridos en Neonatos $(35.5 \mathrm{mg} / \mathrm{dl}$ ) y colesterol VLDL $38.3 \mathrm{mg} / \mathrm{dl} \mathrm{del}$ lado neonatal con el lado placentario $(52.4 \mathrm{mg} / \mathrm{dl}$ y 42.2 respectivamente), posiblemente los lípidos placentarios sean mayores comparados con los del niño, como lo muestra la gráfica 1 dadas las funciones hormonales placentarias con alto depósito lipídico como la producción de estrógenos para favorecer el crecimiento de los órganos sexuales de la madre y de algunos tejidos del feto; y progesterona para promover un desarrollo especial del endometrio y el desarrollo de algunos tejidos y órganos fetales, facilitando el desarrollo del aparato secretor mamario materno (8). No encontramos en la literatura otro estudio que realice esta comparación, lo cual es un aporte valioso para la toma de muestras en investigaciones básico-clínicos o diagnósticas de esta índole.

\section{BIBLIOGRAFIA}

1. Osorio JH. \& Satizábal JM. Efecto del estrés al nacimiento sobre el perfil lipídico del neonato. Rev. Asoc. Col. Nutrición Clínica. 1998. En impresión.

2. Daffos F, Capella-Paulovsky M. and Forrestier F. A new procedure for fetal blood sampling in utero: Preliminary result of fifty three cases. Am. J. Obstet. Gynecol. 1983; 16: 985-987.

3. Possonnier MH, Brossard Y, Demederius $N$ et al. Two hundred intrauterine exchange transfusions in severe blood incompatibilities. Am. J. Obstet. Gynecol. 1989; 161: 709-713.

4. Arias F. Guía práctica para el embarazo y el parto de alto riesgo. 2 de. Mosby-Doyma. Bogotá. 1994; 227.
5. American College of Obstetricians and Gynecologist. Utility of umbilical cord blood acid base assesment, committee opinion No. 91 . Washington, ACOG. 1991.

6. Grundy S, Vega G. Role of apoliproteins levels in clinical practice. Arch. Intermed. 1990; 150: 1579-1582.

7. Osorio JH, Quintero JA, Osorio JJ, Meza DF \& Gutiérrez g. Perfil lipídico en sangre de cordón umbilical. Rev. Col. Obst. Ginecología 1998. En impresión. 